\title{
CCD astrometry of faint compact extragalactic radio sources
}

\author{
I. First results and description of the program \\ E. Costa and P. Loyola \\ Departamento de Astronomía, Universidad de Chile, Casilla 36-D Santiago, Chile
}

Received March 31; accepted July 5, 1999

\begin{abstract}
Optical positions relative to the Hipparcos Catalogue (ESA 1997) have been obtained for the optical counterparts of 15 faint $(B \sim 20-23)$ southern compact extragalactic radio sources (CERS). Most of these sources are not adequately observable (or simply not visible) by means of direct photography with a conventional widefield telescope, so the positions were determined using a multi-step procedure involving CCD and photographic observations, a method which responded both to the need to attain small rms errors and the need to refer the positions to a standard astrometric system. Estimated precisions as good as 60 mas were achieved. For five of the above objects, namely 0936-853, 1221-829, 1604-333, $1647-296$ and $1706+006$, we are proposing a new optical identification.

A comparison with VLBI radio positions available for these sources is presented. The residuals obtained are in most cases consistent with the precision of our optical data, and provide an evaluation of these faint objects as possible radio/optical link sources (or their identification as astrophysically interesting astrometric outliers). Twelve of the CERS observed are either defining or are candidate fiducial objects of the present realization of the International Celestial Reference System (ICRS, Ma et al. 1998), so the optical data obtained for them is potentially useful to help maintain and possibly improve the current link of the Hipparcos reference frame to the ICRS.
\end{abstract}

Key words: astrometry — reference systems

\section{Introduction}

The advent of the Hipparcos Catalogue, which was constructed to coincide with the ICRF (Kovalewsky et al. 1997), and therefore can be considered an extension to the optical domain of the extragalactic radio reference frame, has provided the appropriate framework for the interpretation of subtle positional differences between the radio and optical emission centroids of CERS with star-like optical counterparts (se e.g. Costa \& Loyola 1998).

This type of limited structure analysis is important for identifying the most suitable objects to fine-tune the link between the radio and optical reference frames, and also to uncover astrometric outliers that are potentially interesting sources from an astrophysical point of view. Less directly, the observation of the optical counterparts of CERS with respect to Hipparcos stars provides the means to maintain, and possibly improve, the current link between the Hipparcos reference frame and the ICRS. Although at the moment the above link procedure is less precise than other methods that contributed to the realization of the present link (see e.g. Kovalewsky et al. 1997), on account of the large number of well distributed link sources involved, it has a great potential (see e.g. Zacharias et al. 1995).

With the above objectives in mind, we have started a program to identify the optical counterparts, and determine precise positions with respect to the Hipparcos frame, of faint $(B \sim 20-23)$ CERS. The present survey can be considered an extension to fainter magnitudes of a two decades old CERS monitoring program carried out at the Estación Astronómica de Cerro El Roble (EACR), operated by the University of Chile (see e.g. Costa \& Loyola 1997). In this paper we present our first results and a full description of the project.

Targets were selected from the NRL/USNO proposed reference frame list (Fey 1994) and the list of Jauncey et al. (1989). In order to fully evaluate the capabilities of the multi-step procedure employed to derive the positions, a variety of targets were observed, among them suspected empty fields, CERS with very faint provisional optical identification and CERS with optical counterparts showing evidence of structure. 


\section{Observations}

Optical positions for the CERS were obtained by means of a hybrid multi-step procedure involving CCD and photographic observations, which responds both to the need for attaining usefully small rms errors at very faint magnitudes, and the need to refer the positions to a standard reference system.

On account of their faintness, most of the objects in our program cannot be adequately observed by means of direct photography with wide-field astrographs, so the use of CCD detectors is necessary. However, due to the limited size of the existing CCD chips, and the low density of present fundamental catalogues, CCD astrometry by itself produces only relative positions. Both CCD and wide-field photographic observations are therefore needed; the first for very precise astrometry in the local field of the targets, and the second to link the results to a standard system.

\subsection{Photographic observations}

These observations were carried out with the flat-field $70 / 100 / 210 \mathrm{~cm}$ Maksutov Astrograph (field: $5^{\circ} \times 5^{\circ}$, scale: $99.4^{\prime \prime} / \mathrm{mm}$ ) at the EACR.

Two forming gas hypersensitized Kodak IIIaJ plates, one long exposure (50 min) and one short exposure (2 min), were taken centered on each CERS; both on the same night, and near culmination. A Schott GG385 filter was used throughout. Although not ideal, the use of the blue bandpass was dictated by emulsion availability.

As a first step we identify in the short exposure plate all stars from the Hipparcos Catalogue (which was adopted as primary reference frame) present in the $5^{\circ} \times 5^{\circ}$ field around the CERS. Excluding bright stars, close pairs and stars near the edges of the plates, roughly 60 homogeneously distributed measurable catalogue stars were identified on average in the fields of the sources. We then select about 30 well distributed stars with a magnitude $B \sim 12-14$ in the central $\sim 1.5^{\circ} \times 1.5^{\circ}$ field around the source. The positions of these latter stars are then determined with respect to the stars of the Hipparcos Catalogue, which leads to the establishment of a secondary reference system to be used for position determinations on the long exposure plate. Because of their magnitude, these secondary stars are adequately measurable both in the short and in the long exposure plates (this is not the case of the much brighter primary stars from the Hipparcos Catalogue, which are well overexposed in the deep plates).

In the second step of the procedure we identify all non saturated stars in the $\sim 8.5^{\prime} \times 8.5^{\prime}$ field of the CCD image, which can at the same time be adequately measured on the long exposure plate. They turn out to be stars roughly in the magnitude range $B \sim 15-18$. On average, 20 stars satisfying the above conditions could be identified in the fields of the CCD images. The positions of these stars are then measured with respect to the previously established secondary frame and this leads to the definition of a tertiary reference system which can be used for position determinations on the CCD image.

It should be noted that, since the Hipparcos Catalogue was constructed to coincide with the ICRS, our ad-hoc secondary and tertiary reference frames are tied to the ICRS. The positions of the CERS determined with respect to the tertiary stars in the field of the CCD image will therefore be obtained in the system of the ICRS.

\section{2. $C C D$ observations}

The CCD observations were carried out with the $1.5 \mathrm{~m}$ telescope at Cerro Tololo Interamerican Observatory (CTIO) and with the $2.5 \mathrm{~m}$ telescope at Las Campanas Observatory (LCO). Both telescopes provide a very similar set-up in terms of scale and field: $0.24^{\prime \prime} /$ pixel, $8.19^{\prime} \times 8.19^{\prime}$, CTIO and $0.26^{\prime \prime} /$ pixel, $8.87^{\prime} \times 8.87^{\prime}$, LCO . In both cases the CCD detectors used were Tektronix $2048 \times 2048$ chips with $24 \mu$ pixels. The small scales not only allow an optimum definition of the centroids of the Point Spread Functions (PSF), but also make it possible in a few cases to investigate the optical morphology of the CERS.

Four frames were typically obtained of each target, which were registered and then combined to produce a single work image (see next section). Extensive testing showed that $600 \mathrm{~s}$. exposures (CTIO; $300 \mathrm{~s}$. LCO) were necessary to obtain a good signal to noise ratio for the sources, without saturating the tertiary reference stars. A Johnson $B$ filter was employed throughout for consistency with the photographic observations. All observations were made during culmination to minimize refraction effects, and only with good seeing ( $\left.F W H M \leq 1.5^{\prime \prime}\right)$. In optimum seeing conditions $\left(F W H M \leq 1.0^{\prime \prime}\right)$ we estimate that the limit of detection of our survey is $B \sim 24$.

In all but one case the CCD and the photographic observations were secured at reasonably similar epochs, ensuring a negligible error contribution from the unknown proper motions of the intermediate reference stars. Table 1 gives the epochs of the observations together with information related to the identification of the sources. CT indicates that the CCD observations were carried out at CTIO; LC that they were carried out at LCO. Finding charts for the newly identified optical counterparts (Figs. 1 to 7 ) were produced from their corresponding combined CCD images. The approximate $B$ magnitudes given for them in Table 1 (and those given for sources that were detected to have varied considerably) were estimated from their signal to noise ratio on the combined CCD frames. An asterisk in the Remarks column indicates that additional comments are made in Sect. 6 (Notes on individual objects). 
Table 1. Identification of the sources

\begin{tabular}{|c|c|c|c|c|c|c|c|}
\hline $\begin{array}{c}\text { IAU } \\
\text { Designation }\end{array}$ & $\begin{array}{l}\text { Plate } \\
\text { Epoch }\end{array}$ & $\begin{array}{l}\text { CCD } \\
\text { Epoch }\end{array}$ & & $\begin{array}{c}\text { Approx. } \\
\text { Magnitude }\end{array}$ & Nature & $\begin{array}{c}\text { Finding } \\
\text { Chart }\end{array}$ & Remarks \\
\hline $0826-373$ & 97.04 .04 & 95.04 .24 & CT & & $\mathrm{EF}$ & & \\
\hline $0931-114$ & 97.04 .05 & 97.02 .07 & CT & & $\mathrm{EF}$ & & \\
\hline & & 95.05 .06 & $\mathrm{LC}$ & & & & \\
\hline $0936-853$ & 97.04 .03 & 95.04 .23 & CT & 21 & QSO? & 1 & * \\
\hline $1034-058$ & 97.04 .06 & $\begin{array}{l}97.02 .07 \\
95.05 .06\end{array}$ & $\begin{array}{l}\mathrm{CT} \\
\mathrm{LC}\end{array}$ & & $\mathrm{EF}$ & & \\
\hline $1048-313$ & 96.05 .13 & 95.04 .22 & $\mathrm{CT}$ & 18.5 & QSO? & 1,3 & * \\
\hline $1133-681$ & 96.05 .14 & 95.04 .21 & $\mathrm{CT}$ & 22.5 & QSO? & 4 & * \\
\hline $1213-172$ & & & $\mathrm{CT}$ & & & & * \\
\hline $1221-829$ & 96.05 .12 & 95.04 .23 & $\mathrm{CT}$ & 22 & QSO? & 1 & * \\
\hline $1234-504$ & 95.06 .21 & 95.04 .24 & CT & & $\mathrm{EF}$ & & \\
\hline $1251-713$ & 96.05 .13 & $\begin{array}{l}95.04 .23 \\
95.05 .06\end{array}$ & $\begin{array}{l}\text { CT } \\
\mathrm{LC}\end{array}$ & 21.5 & QSO? & 4 & \\
\hline $1313-333$ & 91.04 .17 & 95.04 .22 & $\mathrm{CT}$ & var. & QSO & 5 & * \\
\hline $1334-649$ & 96.05 .14 & 95.04 .25 & $\mathrm{CT}$ & & $\mathrm{EF}$ & & \\
\hline $1443-162$ & 95.07 .22 & 95.04 .22 & $\mathrm{CT}$ & 20.5 & QSO? & 1,3 & $*$ \\
\hline $1555-140$ & & & CT & & & 2 & * \\
\hline $1602+014$ & 96.05 .13 & $\begin{array}{l}95.04 .21 \\
95.05 .08\end{array}$ & $\begin{array}{l}\text { CT } \\
\text { LC }\end{array}$ & 20.5 & AGN & 4 & \\
\hline $1604-333$ & 96.05 .11 & 95.04 .23 & $\mathrm{CT}$ & 18 & QSO? & 1 & $*$ \\
\hline $1647-296$ & 95.06 .20 & $\begin{array}{l}95.04 .24 \\
95.05 .07\end{array}$ & $\begin{array}{l}\text { CT } \\
\text { LC }\end{array}$ & 21 & QSO? & 1 & * \\
\hline $1706-174$ & 95.07 .21 & 95.04 .25 & $\mathrm{CT}$ & 22 & QSO? & 1 & * \\
\hline $1706+006$ & 94.08 .04 & $\begin{array}{l}95.04 .21 \\
95.05 .08\end{array}$ & $\begin{array}{l}\text { CT } \\
\text { LC }\end{array}$ & 21.5 & AGN & 4 & $*$ \\
\hline $1740-517$ & 95.08 .26 & 95.04 .24 & $\mathrm{CT}$ & & EF? & & * \\
\hline $1813-241$ & 96.09 .04 & 95.04 .23 & $\mathrm{CT}$ & & $\mathrm{EF}$ & & \\
\hline $1815-553$ & 94.06 .09 & 95.04 .22 & $\mathrm{CT}$ & 21 & QSO? & 4 & * \\
\hline $1908-201$ & 95.07 .20 & 95.04 .25 & $\mathrm{CT}$ & 19.5 & QSO? & 6 & $*$ \\
\hline $1925-610$ & 95.08 .26 & 95.04 .22 & CT & 20.5 & QSO? & 4 & * \\
\hline
\end{tabular}

1 indicates a newly identified optical counterpart, LC stands for Las Campanas,

CT stands for Cerro Tololo.

\author{
References to finding charts \\ [1] This paper \\ [2] Bolton J.G., Shimmins A.J., Wall J.V., 1975, A.J.P. Astrophys. Supp. 34, 1 \\ [3] Drinkwater M.J., Webster E.L., Francis P.J., et al., 1997, MNRAS 284, 85 \\ [4] Jauncey D.L., Savage A., Morabito D.D., et al., 1989, AJ 98, 54 \\ [5] Jauncey D.L., Batty M.J., Gulkis S., et al., 1982, AJ 87, 763 \\ [6] Torres C., Wroblewski H., 1987, A\&AS 69, 23
}

\section{Reductions}

The $X, Y$ coordinates of the reference stars on the photographic plates were measured to $1 \mu\left(0.1^{\prime \prime}\right.$ at the plate scale) with a digital Zeiss-Jena Ascorecord measuring machine. Only one setting was made on the catalogue, secondary and tertiary reference stars. In the few cases in which the optical counterpart of the CERS was measurable directly on the long exposure plate, at least 4 settings were made on it. The photographic position thus determined was used to check for consistency. Plates were measured both in direct and reverse positions in an effort to cancel possible systematic errors in the $X, Y$ values. Six term (const., $X, Y, X Y, X^{2}, Y^{2}$ ) quadratic relations were used in the reductions. Third order terms were not included because the flat field of the Maksutov Astrograph is almost free of distortions. Also, since all plates were taken near culmination, refraction third order terms were not important. The standard deviation of the differences 
N

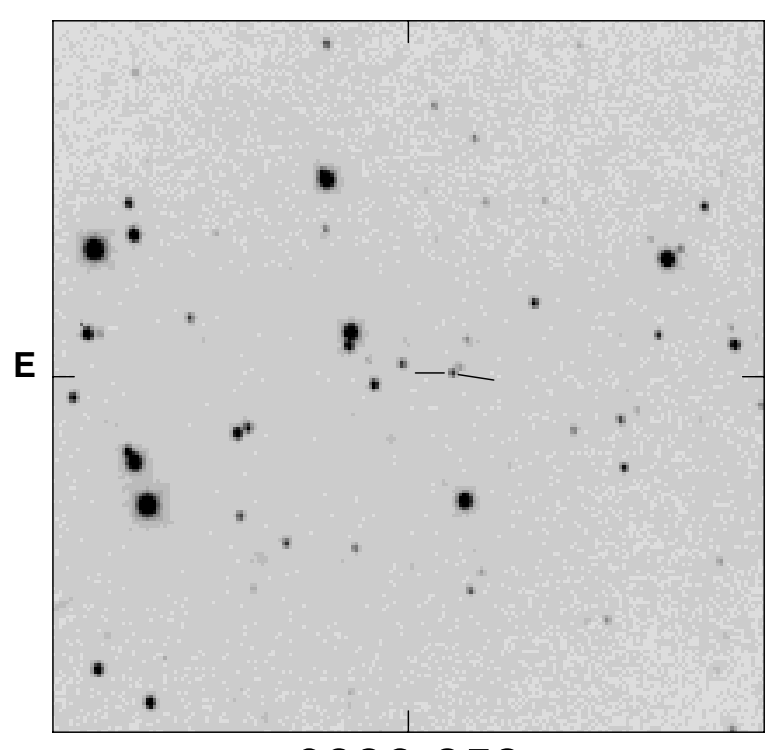

0936-853

Fig. 1.

Figures 1-7: Finding charts for the newly identified optical counterparts, as indicated in Table 1. Charts are 4.1 arcmin on a side. See Sect. 6 for details

between calculated and catalogue values of the Hipparcos stars turned out to be $0.23^{\prime \prime}$ in $X$ and $0.22^{\prime \prime}$ in $Y$.

All raw CCD frames were first calibrated (overscan, zero level and flat field corrected) using standard IRAF (version 2.10, NOAO, University of Arizona) tasks. The four CCD images taken of each CERS were then registered and combined using algorithms available in the IRAF IMAGES package, to produce the final image from which the $X, Y$ coordinates of the PSF centroids of the CERS and the tertiary stars were extracted. This latter step was carried out by means of the PEAK task within the DAOPHOT package (Stetson 1987). Although this package was originally designed for photometric programs, it has proven to be an excellent tool to compute image positions (Monet 1992; Anguita \& Loyola 1995).

\section{Results}

The resulting optical positions with respect to the Hipparcos frame, and therefore in the system of the ICRS, are given in Table 2. The first column gives the IAU designation of the sources, the second and fourth columns their right ascensions and declinations, and the third and fifth columns their corresponding total internal errors. The positions based on CCD observations made at CTIO are identified as CT in the Remarks column; those based on CCD observations made at LCO as LC. The positions derived from direct photography are identified as Phot.

\section{Errors}

\subsection{Total internal errors}

The total internal errors $\epsilon_{\mathrm{T}}$ of the positions of the sources, given in Cols. 3 and 5 of Table 1 , were estimated by means of the relation: $\epsilon_{\mathrm{T}}^{2}=\epsilon_{\mathrm{r}, \mathrm{ccd}}^{2}+\left(\epsilon_{\mathrm{t}, \mathrm{ccd}}^{2}+\bar{\epsilon}_{\mathrm{t}}^{2}\right) \Sigma D_{\mathrm{r}, \mathrm{ccd}}^{2}$, in which $\epsilon_{\mathrm{r}, \mathrm{ccd}}$ and $\epsilon_{\mathrm{t}, \mathrm{ccd}}$ are the errors in the determination of the source and tertiary stars image centroids positions on the CCD frame, respectively, and $\bar{\epsilon}_{\mathrm{t}}$ is the mean value of the total internal errors of the positions determined for the tertiary stars on the long exposure plate. $\Sigma D_{\text {r,ccd }}^{2}$ is the sum of the squares of the dependences (Schlesinger 1911) for the source on the CCD frame. $\bar{\epsilon}_{\mathrm{t}}$ was estimated by means of the analogous relation: $\bar{\epsilon}_{\mathrm{t}}^{2}=\epsilon_{\mathrm{t}, 1}^{2}+\left(\epsilon_{\mathrm{s}, 1}^{2}+\bar{\epsilon}_{\mathrm{s}}^{2}\right) \overline{\Sigma D_{\mathrm{t}, 1}^{2}}$, in which $\epsilon_{\mathrm{t}, 1}$ and $\epsilon_{\mathrm{s}, 1}$ are the errors in the measurement of the tertiary and secondary stars positions on the long exposure plate, respectively, and $\bar{\epsilon}_{\mathrm{s}}$ is the mean value of the total internal errors of the positions determined for the secondary reference stars on the short exposure plate. $\overline{\Sigma D_{\mathrm{t}, 1}^{2}}$ is the mean value of the sum of the squares of the dependences for the tertiary stars on the long exposure plate. Finally, $\bar{\epsilon}_{\mathrm{s}}$ was in turn estimated from: $\bar{\epsilon}_{\mathrm{s}}^{2}=\epsilon_{\mathrm{s}, \mathrm{s}}^{2}+\left(\epsilon_{\mathrm{p}, \mathrm{s}}^{2}+\epsilon_{\mathrm{C}}^{2}\right) \overline{\Sigma D_{\mathrm{s}, \mathrm{s}}^{2}}$, in which $\epsilon_{\mathrm{s}, \mathrm{s}}$ and $\epsilon_{\mathrm{p}, \mathrm{s}}$ are the errors in the measurement of the secondary and primary (catalogue) star positions on the short exposure plate, respectively, and $\epsilon_{\mathrm{C}}$ is the total error of the Hipparcos Catalogue in the corresponding zone of the sky. $\overline{\Sigma D_{\mathrm{s}, \mathrm{s}}^{2}}$ is the mean value of the sum of the square of the dependences for the secondary stars on the short exposure plate. Although it was predictable that the error contribution of the Hipparcos Catalogue $\left(\epsilon_{\mathrm{C}}\right.$, which comprises the error in the positions and the errors accumulated due to uncertainties in the proper motions) would be negligible compared to the measurement errors, we nevertheless did not use mean values, and calculated $\epsilon_{\mathrm{C}}$ on a zonal basis. It was determined that $\epsilon_{\mathrm{C}}(\alpha)$ and $\epsilon_{\mathrm{C}}(\delta)$ varied between 4.7 and 10 mas, and 1.1 and 8.9 mas, respectively.

The total internal errors of the positions derived from direct photography were estimated by means of the relations given in Sect. 4.1 of Costa \& Loyola (1992).

\subsection{Measurement errors}

The errors in the measurement of the catalogue, secondary and tertiary reference stars positions on the photographic plates used in the above relations were average values previously determined, this because in practice only one setting was made on all reference stars. The values adopted were $\epsilon_{\mathrm{p}, \mathrm{s}}(X)= \pm 0.19^{\prime \prime}, \epsilon_{\mathrm{p}, \mathrm{s}}(Y)= \pm 0.18^{\prime \prime}$, $\epsilon_{\mathrm{s}, \mathrm{s}}(X)= \pm 0.10^{\prime \prime}, \epsilon_{\mathrm{s}, \mathrm{s}}(Y)= \pm 0.11^{\prime \prime}, \epsilon_{\mathrm{s}, \mathrm{l}}(X)= \pm 0.13^{\prime \prime}$ $\epsilon_{\mathrm{s}, 1}(Y)= \pm 0.14^{\prime \prime}, \epsilon_{\mathrm{t}, 1}(X)= \pm 0.12^{\prime \prime}$ and $\epsilon_{\mathrm{t}, \mathrm{l}}(Y)= \pm 0.13^{\prime \prime}$.

As error in the determination of the tertiary stars image centroid positions on each combined CCD frame, $\epsilon_{\mathrm{t}, \mathrm{ccd}}$, we adopted the standard deviation of the mean of 
Table 2. Optical positions in the ICRS system of 15 compact extragalactic radio sources

\begin{tabular}{|c|c|c|c|c|c|}
\hline$(1)$ & $\overline{(2)}$ & $\overline{(3)}$ & $(4)$ & $(5)$ & $(6)$ \\
\hline IAU & RA & $\epsilon_{\alpha} \cos \alpha$ & DEC & $\epsilon_{\delta}$ & Remarks \\
\hline Designation & $\mathrm{h} \mathrm{m} \mathrm{s}$ & $\pm^{\prime \prime}$ & 0111 & $\pm^{\prime \prime}$ & \\
\hline $0936-853$ & 093032.630 & 0.07 & -853359.37 & 0.09 & $\mathrm{CT}$ \\
\hline \multirow[t]{2}{*}{$1048-313$} & 105104.773 & 0.07 & -313814.31 & 0.07 & $\mathrm{CT}$ \\
\hline & 04.763 & 0.10 & 14.45 & 0.13 & Phot. \\
\hline $1133-681$ & 113602.121 & 0.10 & -682706.01 & 0.08 & $\mathrm{CT}$ \\
\hline $1221-829$ & 122454.392 & 0.08 & -83 1309.53 & 0.09 & $\mathrm{CT}$ \\
\hline \multirow[t]{2}{*}{$1251-713$} & 125459.967 & 0.09 & -713818.21 & 0.08 & $\mathrm{CT}$ \\
\hline & 59.962 & 0.07 & 18.21 & 0.06 & $\mathrm{LC}$ \\
\hline \multirow[t]{2}{*}{$1313-333$} & 131608.003 & 0.08 & -333859.19 & 0.07 & $\mathrm{CT}$ \\
\hline & 08.010 & 0.23 & 59.11 & 0.12 & Phot. \\
\hline $1443-162$ & 144553.385 & 0.06 & -162901.67 & 0.06 & $\mathrm{CT}$ \\
\hline \multirow[t]{2}{*}{$1602+014$} & 160445.336 & 0.09 & +011750.44 & 0.07 & $\mathrm{CT}$ \\
\hline & 45.372 & 0.06 & 50.37 & 0.07 & $\mathrm{LC}$ \\
\hline \multirow[t]{2}{*}{$1604-333$} & 160734.817 & 0.06 & -333108.97 & 0.06 & $\mathrm{CT}$ \\
\hline & 34.816 & 0.20 & 09.16 & 0.17 & Phot. \\
\hline \multirow[t]{2}{*}{$1647-296$} & 165039.554 & 0.07 & -294347.10 & 0.07 & $\mathrm{CT}$ \\
\hline & 39.534 & 0.08 & 47.11 & 0.07 & $\mathrm{LC}$ \\
\hline $1706-174$ & 170934.324 & 0.08 & -172853.75 & 0.09 & $\mathrm{CT}$ \\
\hline \multirow[t]{2}{*}{$1706+006$} & 170844.636 & 0.11 & +003509.02 & 0.12 & $\mathrm{CT}$ \\
\hline & 44.605 & 0.08 & 09.07 & 0.09 & $\mathrm{LC}$ \\
\hline $1815-553$ & 181945.395 & 0.10 & -552120.63 & 0.07 & $\mathrm{CT}$ \\
\hline $1908-201$ & 191109.657 & 0.07 & -200655.20 & 0.06 & $\mathrm{CT}$ \\
\hline \multirow[t]{2}{*}{$1925-610$} & 193006.161 & 0.08 & -605609.31 & 0.09 & $\mathrm{CT}$ \\
\hline & 06.193 & 0.16 & 09.29 & 0.15 & Phot. \\
\hline
\end{tabular}

CT stands for Cerro Tololo,

LC stands for Las Campanas,

Phot. indicates a position derived from direct photography.

the $X, Y$ positions of these stars in the four individual frames that were used to produce the combined CCD image for each target, from which their final $X, Y$ positions were determined. $\epsilon_{\mathrm{t}, \mathrm{ccd}}$ varied between 3 and 25 mas in $X$, and between 3 and 13 mas in $Y$.

The same method was used to estimate the error in the determination of the source image centroid position on each combined CCD frame, $\epsilon_{\mathrm{r}, \mathrm{ccd}}$. It should be noted however, that because of the extreme faintness of some of the optical counterparts, their PSF were clearly less well defined in the individual frames compared to the combined image (this was not the case for the much brighter tertiary stars). For this reason, we consider that this procedure yields in general an upper limit of $\epsilon_{\mathrm{r}, \mathrm{ccd}}$. This error varied between 4 and 80 mas in $X$, and between 3 and 90 mas in $Y$.

For those cases in which the CERS was visible on the long exposure plate, the standard deviation of the mean of multiple settings made on the optical counterpart was adopted as the measurement error. This error varied between 70 and 220 mas in $X$, and between 110 and 160 mas in $Y$.

In any case, it must be kept in mind that all of the above Sigmas are based on only four (and in a few cases on only three) independent measures.

\section{Notes on individual objects}

0936-853: No optical identification was available for this object. Figure 1 shows the optical counterpart we propose. In wide-field photographic plates, this object has an elongated image due to a very close star. On the CCD images it is clearly resolved.

1048-313: Drinkwater et al. (1997) independently identified the same optical counterpart for this source. Here we present an improved finding chart (Fig. 2).

1213-172: A bright star near the source prevented CCD imaging. No optical counterpart is known for this source. 1221-829: The provisional $m \sim 22.5$ optical identification proposed by Jauncey et al. (1989) is incorrect. The correct optical counterpart is an object of similar brightness that lies to the NE, as shown by Fig. 3 .

1313-333: Large inconsistencies detected in the value assigned to its magnitude suggest that this source is grossly variable in the optical.

1443-162: Same situation as 1048-313. See Fig. 4.

1555-140: The optical counterpart of this object is a bright and diffuse galaxy. Although different integration times were tested during CCD imaging, none of them produced a point-like nucleus appropriate for astrometry. 1604-333: No optical counterpart was known for this source. Figure 5 shows the identification we are proposing. 
N

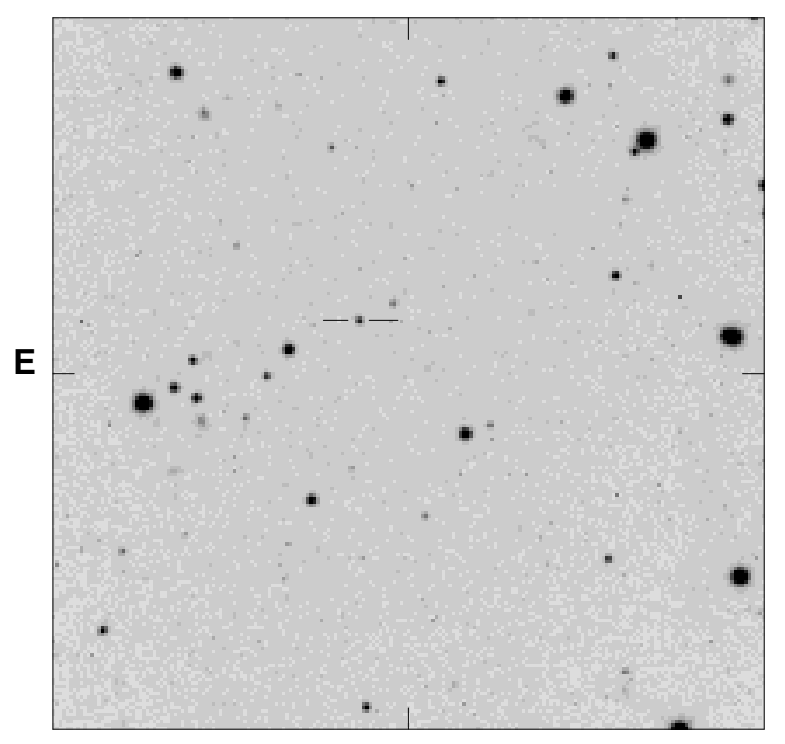

$1048-313$

Fig. 2.

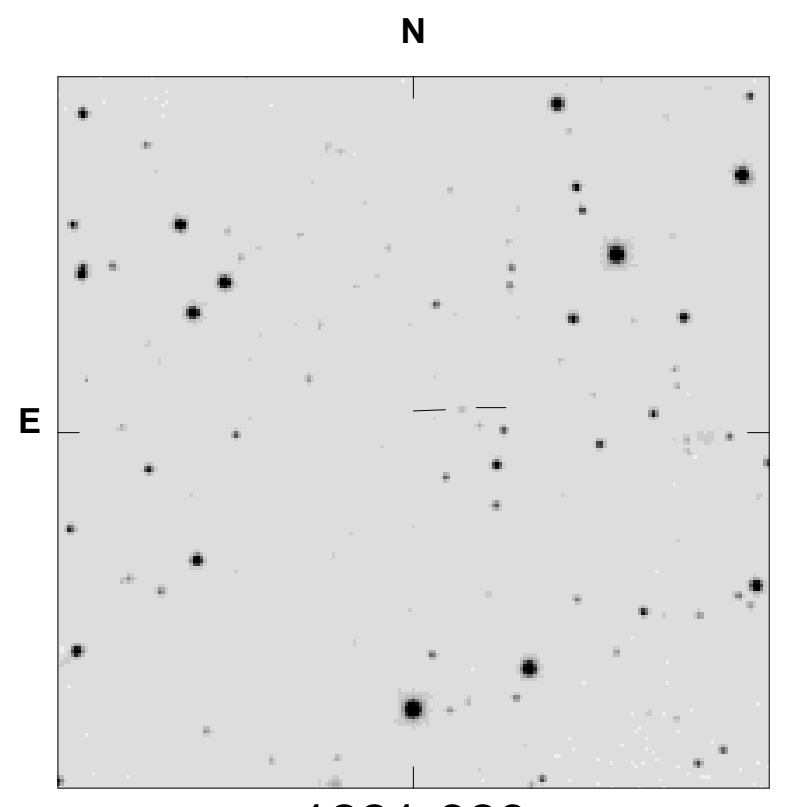

$1221-829$

Fig. 3.

On account of the fairly large RA radio-optical residual derived (see Table 3 ) it should be considered provisional. In any case, we found no evidence of an alternative fainter counterpart.

1647-296: No optical identification was known of this source. Figure 6 shows the identification we propose. The optical object is a close pair. It is just resolved on the CCD frames, but it looks like a single image in the wide-field photographic plates. The faintest (in $B$ ) of the pair is the correct optical counterpart.

1706-174: The $m \sim 17.5$ QSO proposed by Johnston

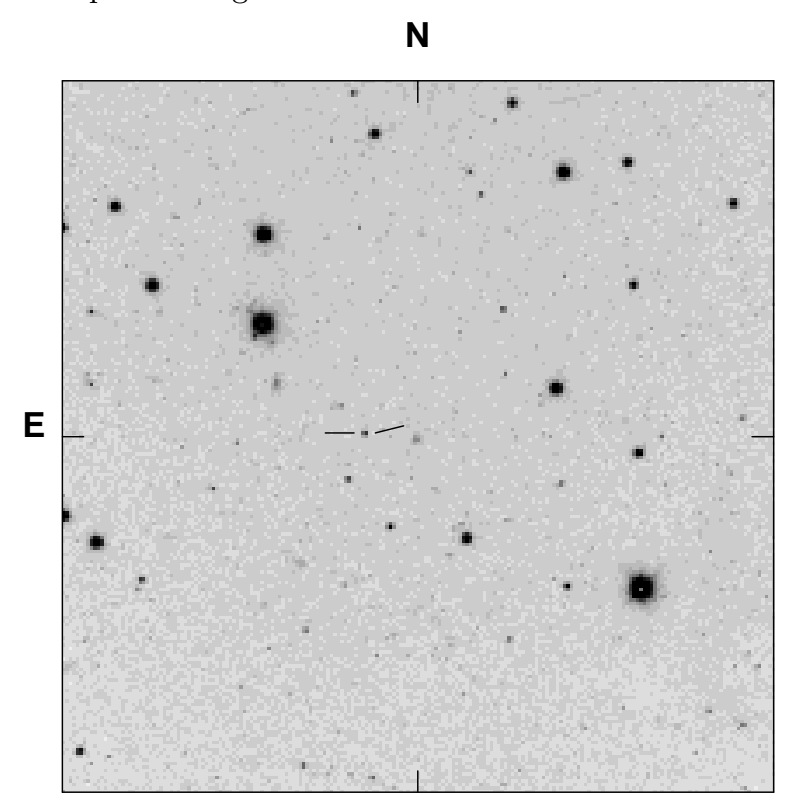

$1443-162$

Fig. 4 .

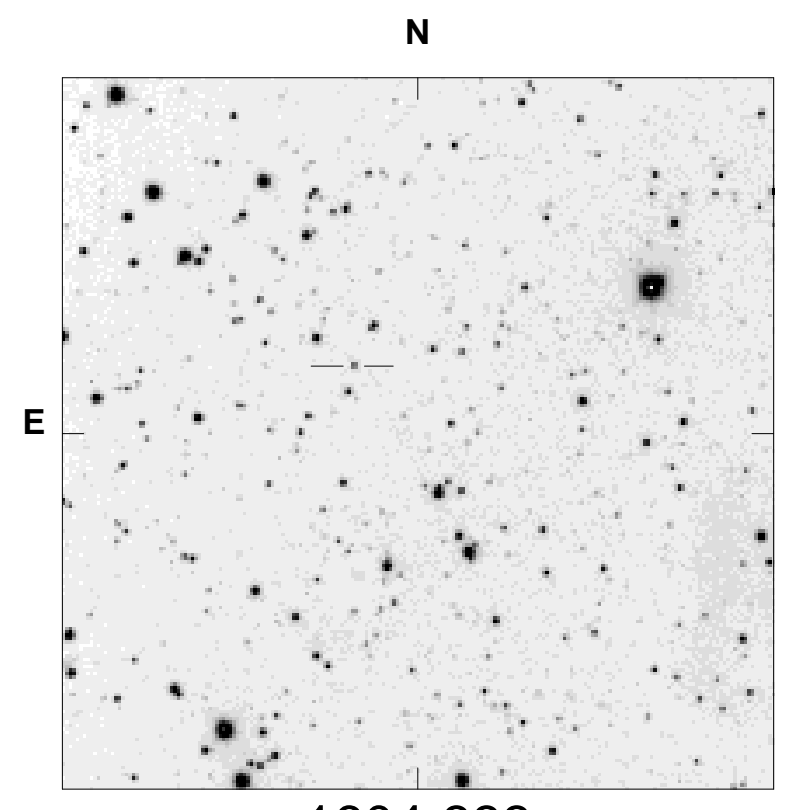

$1604-333$

Fig. 5 .

et al. (1995) as optical identification of this source is incorrect (no finding chart was published). Here we propose a much fainter optical counterpart $(B \sim 22)$, as shown in Fig. 7.

1706+006: Differences detected in the magnitude assigned to this object suggest that it is optically variable.

1740-517: Empty field? At the limit of detection an object seems to be present in the radio source position.

1815-553: Optically variable. This object was roughly two magnitudes brighter when observed by Jauncey et al. (1989). 
$\mathbf{N}$

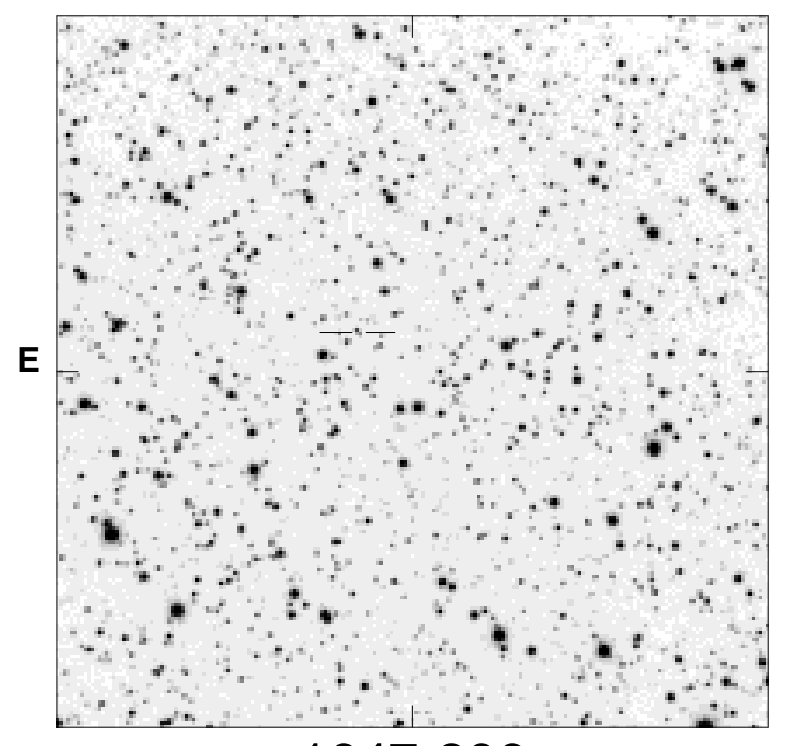

$1647-296$

Fig. 6.

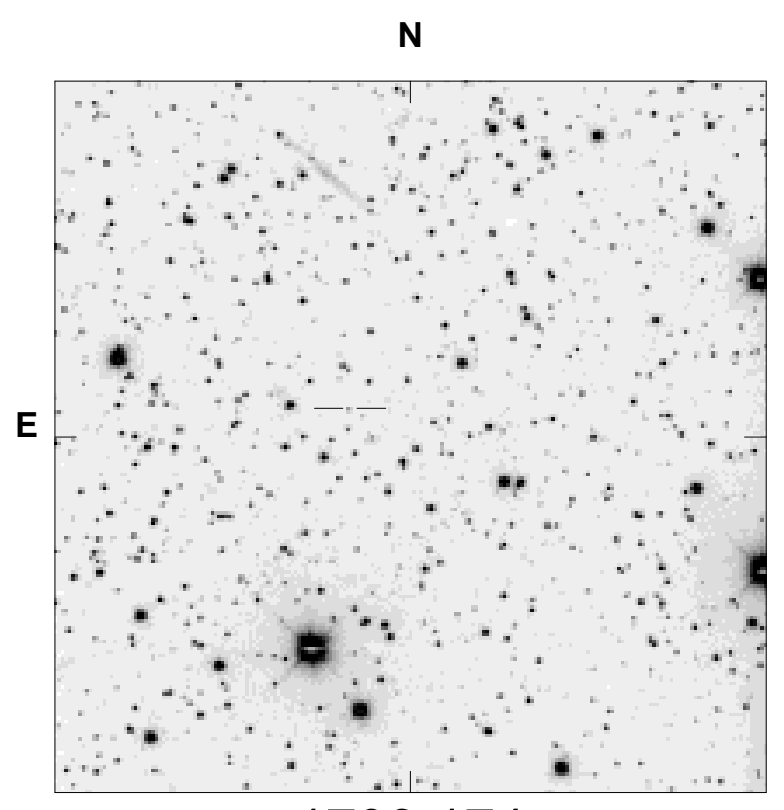

$1706-174$

Fig. 7.

1908-201: Not the $m \sim 22$ optical identification proposed by Johnston et al. (1995). Torres \& Wroblewski (1987) identified a much brighter object as the optical counterpart, but gave a wrong value for its magnitude. Here we confirm this latter identification and give a new estimation of its magnitude.

1925-610: Optically variable. This object brightened roughly two magnitudes in the timespan (4 months) between our CCD and photographic observations. The magnitude given is an average of both estimations.
Table 3. Comparison in the ICRS with the VLBI radio positions given by Ma et al. (1998). See Sect. 7 for details

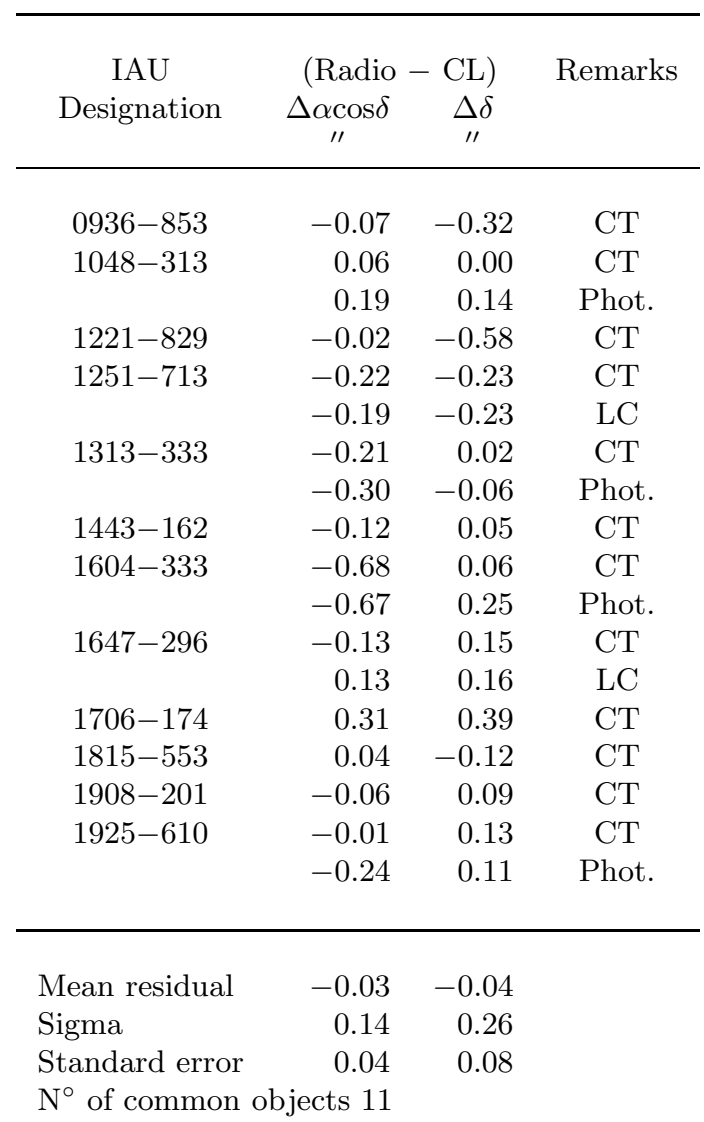

CT and LC stand for Cerro Tolo and Las Campanas respectively.

Phot. indicates a comparison with a position derived from direct photography.

\section{Discussion}

Our results show that combining CCD observations with conventional photographic astrometry, it is possible to obtain "absolute" (i.e. refered to a standard astrometric system) optical positions of very faint $(B \sim 20-23)$ CERS with a precision level as good as 60 mas.

Although the ultimate precision attainable with our method is hampered by the accuracy with which the positions of the reference stars can be determined by measuring the plates with a conventional manual measuring device, an important reduction of the total internal error results from the significant decrease of $\epsilon_{\mathrm{r}}$, which completely dominates the total internal error in a purely photographic approach (this is true even in the case of moderately bright $(B \sim 19.5)$ objects; see Costa \& Loyola 1992).

It should be stressed furthermore that the method proposed is also capable of precise astrometry of objects that are not measurable -or simply not visible- on a wide-field telescope plate, and is very effective at exploring suspected 
empty fields in search of faint optical counterparts (for roughly $50 \%$ of the empty fields explored we are proposing a new optical identification).

In Table 3 we present a comparison in the ICRS with the VLBI radio positions given by Ma et al. (1998), in the sense radio minus this work (CL). To calculate the mean differences presented at the bottom of the table we considered only optical positions derived from CCD observations. The statistics are based on only 11 objects; this because source 1604-333 has a right ascension radio-CL residual three times the standard deviation of the mean of the complete sample. Keeping in mind that the errors in the measurement of the primary, secondary and tertiary reference stars used to calculate the total internal errors of our positions were average values based on previous experience (see Sect. 5) -which opens the possibility that for some objects these errors may have been underestimatedthe residuals obtained are consistent with the estimated precision of our optical data.

Since our optical positions are displayed in essentially the same reference system as the radio positions, the radio-CL differences given in Table 3 provide an evaluation of these objects as possible radio/optical frame link sources (or their identification as astrophysically interesting astrometric outliers); in five cases (namely 0936-853, $1221-829,1604-333,1647-296$ and $1706-174$ ) the first one available. A few objects show large residuals which are not consistent with the estimated errors of their positions presented in Table 2. These cases are difficult to explain; they could be astrometric outliers, or, as explained in the above paragraph, their positional errors may have been underestimated. Also, we cannot rule out the existence of an undetected magnitude equation.

Considering that 12 of the CERS observed are either defining or candidate fiducial radio sources of the present realization of the ICRS, our optical data is potentially useful to help maintain, and possibly improve, the current link of the Hipparcos reference frame to the ICRS.

Acknowledgements. We are indebted to Cerro Tololo Interamerican Observatory for donating the plate material that made possible our survey. We are also indebted to Prof. C. Anguita for his interesting comments, and to $\mathrm{M}$. Wishnjewski and L.E. González for measuring the plates. This work was financed by the Fondo Nacional de Investigación Científica y Tecnológica (proyecto $\mathrm{N}^{\circ} 1970767$ Fondecyt).

\section{References}

Anguita C., Loyola P., 1995 (private communication)

Costa E., Loyola P., 1992, A\&AS 96, 183

Costa E., Loyola P., 1997, A\&AS 122, 441

Costa E., Loyola P., 1998, A\&AS 131, 259

ESA, 1997, "The Hipparcos and Tycho Catalogues", ESA SP-1200, European Space Agency, Vols. 1-17

Fey A.L., 1994, (private communication)

Jauncey D.L., Savage A., Morabito D.D., et al., 1989, AJ 98, 54

Johnston K.J., Fey A.L., Zacharias N., et al., 1995, AJ 110, 880

Kovalewsky J., Lindegren L., Perryman M.A.C., et al., 1997, A\&A 323, 620

Ma C., Arias E.F., Eubanks T.M., et al., 1998, AJ 116, 516

Monet D.G., 1992, "Introduction to CCD Astrometry", Astronomical CCD Observing and Reduction Techniques, ASP Conf. Ser. 25, 221, Howell S.B. (ed.)

Schlesinger F., 1911, ApJ 33, 161

Stetson P.B., 1987, PASP 99, 191

Zacharias N., De Vegt C., Winter L., Johnston K.J., 1995, AJ 110,3093 